\title{
Fall and Rise of Discourse Connectives: A Cross-language Study
}

\author{
Kun Sun \\ Department of Linguistics, University of Tübingen
}

\begin{abstract}
Recent studies have claimed that the amount of discourse connectives or discourse markers has risen in multiple languages. However, the thesis has seldom been evaluated using comprehensive empirical data evidence. This study investigates the historical changes of frequencies in discourse connectives in the Chinese, English, French, German and Spanish over the last two hundred years. We find that the frequencies of the majority of discourse connectives showed a marked decrease in the latter four languages. These research results show that the frequencies of discourse markers in these languages have not in fact increased over the last two centuries. However, an increase of frequencies can be seen after 1980. Our findings are consistent with the changes of rationality in language recently reported. However, we believe that such phenomena are caused by the "colloquialization" trend. The current study can make contributions to a better understanding of the changes in language and their underlying mechanism, and it helps us to draw a bigger picture on evolutionary patterns of human society and human behavior.
\end{abstract}

Keywords: frequency changes; decline trend; implicit discourse relation; historical development; colloquialization 


\section{Introduction}

Discourse connectives are words or phrases connecting or relating discourse units. They are used to indicate discourse relations. A discourse relation is the semantic or logical meaning of the connections between discourse units. Discourse connectives play an important role in creating coherent discourse because they mark the semantic relation between discourse units and then help language users to attain an understanding of textual coherence. Put it simply, discourse connectives play a central role in creating coherent texts. Besides linking sentences or clauses at the surface level, discourse connectives can facilitate the processing of discourse relations by helping comprehenders to infer the intended coherence relation holding between two text spans.

For example, "As he stood on the harbour thinking about his son (1), he wondered if the family would ever get to make the journey(2). However, soon after this disappointment (3) the father discovered how lucky the family had been (4).". In this example, "as" provides the link between clause (1) and (2). This discourse connective allows readers/listeners to know that the two clauses have a temporary relation. Similarly, "soon after" allows readers to know that clause (3) and clause (4) are linked by a temporary relation. Finally, "however" connects the two discourse units made up by (1)-(2) and (3)-(4) respectively and expresses the contrast between them. Without these discourse connectives, it is likely to cause an incoherent text. More seriously, the underlying logical relation from this text may become unclear or vague for listener or reader without them. Pragmatic inference (or effective commun) is not easy to make when the logical relation is not clear.

Such pragmatic inferences happening above sentence level include relations of contrast, contingency, expansion and temporality. They are often facilitated by the use of explicit connectives, which function as processing instructions. This facilitation has been repeatedly evidenced in comprehension and processing studies (Sanders and Noordman, 2000; Van Silfhout et al., 2015). Moreover, a large amount of discourse research has focused on how discource connectives are processed (Torabi Asr and Demberg, 2020; KöhneFuetterer et al., 2021). Through fMRI evidence, Prado et al. (2015) found that that pragmatic processing interacts with logical inference-making when understanding arguments in narrative discourse. Although these discourse connectives also play a logical role in language and they are central to everyday conversation, few studies have been investigated.

Moreover, discourse connectives play a critical role in establishing theoretical discourse analysis and computational discourse studies. For exam- 
ple, one influential discourse corpus, the Penn Discourse Treebank (PDTB) (Webber et al., 2019), uses discourse connectives to annotate English discourse structures. The PDTB uses discourse connectives as heads to form dependencies concerning local discourse structure. In this sense, discourse connectives play an essential role in this corpus. The PDTB has greatly affected theoretical discourse analysis, discourse corpora building and a

Discourse connectives and their relevant concepts (e.g., discourse markers, discourse particles) have been become quite popular (Lenk, 1998; Fraser, 1999) in studies of pragmatics and discourse. The main reason is that these studies have found that discourse connectives provide necessary discourse and pragmatic information in language as mentioned above. This has led to the thesis of "the Rise of Discourse Markers", which has gradually become accepted (Heine et al., 2022). Although discourse connectives are one essential part of discourse markers, this nonetheless gives rise to the illusion that the use of discourse connectives has increased and thus that discourse connectives have risen in frequency. Although frequency effects have been extensively and intensively studied in language variations, language processing and language acquisition (ref. Ellis, 2002; Grainger, 1990). However, the diachronic change of frequency has seldom been investigated to associate with usage of a language phenomenon. If we say that a language phenomenon is on the rise, it implies that language users have been using it more frequently. If its frequency is decreasing, it is difficult to say that it is on the rise. A frequency increase in one language phenomenon at least suggests that there is a motivation for this language phenomenon and that there is an increase in its usage (Sun and Baayen, 2021). In other words, if it is known that the frequencies of discourse connectives have in fact increased significantly, it is plausible to believe in "a rise of discourse connectives". In order to test this hypothesis and the surrounding issues, we need to examine the historical data on discourse connectives, and the historical data on discourse connectives can provide empirical evidence to test this hypothesis.

So far, few has examined historical changes of discourse connectives comprehensively. Most of such historical studies focused on English rather than cross-language studies. Although previous studies have investigated the diachronic changes of frequencies in specific discourse markers, we do not think that such investigations are comprehensive enough to represent all discourse connectives ( Lenker and Meurman-Solin, 2007; Claridge, 2013; Rysová, 2017). In order to see the bigger picture, we will examine the diachronic changes in all discourse connectives derived from large-scale corpora and make a quantitative analysis across different languages. This study is possible thanks to the growing interest in discourse analysis and the avail- 
ability of large-scale corpora.

In addition, recently the tremendous influence of computational approaches has been widespread and rapidly increasing. The quick growth and development of digital humanities reflects how computational approaches have influenced the humanities (Berry, 2011). For instance, "culturomics" uses computational lexicology to study human behavior and cultural trends through the quantitative analysis of digitized texts (Michel et al., 2011). Researchers can mine large digital archives to investigate cultural phenomena as reflected in language and word usage. With the digitization of written language, cultural trend analysis based around methods to extract quantitative patterns from word counts has the potential to provide novel insights into the development of human society. For example, Scheffer et al. (2021) used emotion words/ratiof fact-related words dropped while emotion-laden language surged, a trend paralleled by a shift from collectivistic to individualistic language. The other study (Iliev and Axelrod, 2016) used the similar method to find an increase in the use of causality over history. As discourse connectives represent logical connection and pragmatic inference to some extent, their historical changes also reflect how people employed such logical device to show their cognitive interest in language and communication. We wonder whether the present study will have some connections with them to gain a bigger picture.

The present study looks at the majority of discourse connectives comprehensively from the diachronic point of view and the perspective of crosslanguage investigations. To our knowledge, this is the first time such an investigation has been made. We believe that by drawing upon the data on the historical development of this linguistic phenomenon, the current study can reveal much about general tendencies in the use of present-day discourse connectives and human cognitive interest in discourse. Our research questions are as follows:

1) Have the frequencies of discourse connectives in English increased or declined significantly over the last two centuries?

2) Can we see such similar changes in the other languages (i.e., Chinese, French, German and Spanish)?

3) Do the diachronic changes of frequencies in discourse connectives provide empirical evidence to support the claim concerning a rise of discourse connective? Could these diachronic changes provide us with a bigger picture on evolution of human society? 


\section{Materials and Methods}

First, we need to obtain a comprehensive list of discourse connectives. The release of the PDTB (Webber et al., 2019) provides a high-quality and largescale discourse resource for the English language. Corpora annotated with discourse relations for eight languages were developed following the release of the PDTB. The most recent release is PDTB3.0. This version contains a manual which provides a detailed list of discourse connectives (Webber et al., 2019). In this manual, an individual discourse connective is specified with its different semantic meanings as well as its frequencies. Such data are very important and valuable for historical studies and especially in the present context, given that we want to know the frequencies of different semantic meanings for this specific discourse connective. More importantly, the release of PDTB has been extremely influential because such styles have been adopted to annotate in the other languages, such as Chinese, French and German. By adopting the classification of discourse relationship from PDTB, we can classify discourse connectives into several types according to their meanings. In this case, we could examine the historical changes of each type of these discourse connectives among these languages.

Secondly, after obtaining the list of discourse connectives, we need to capture the data on the historical frequencies for these discourse connectives. The Google Books Corpora (Michel et al., 2011) provide historical data for a number of languages. These Google Books corpora can help us make a crosslanguage study. Google Books corpora provide historical data for Chinese, English, French, German, Hebrew, Italian, Russian and Spanish. Modern Hebrew has been spoken after 1945. When Italy was unified in 1861, the decision was taken to make Tuscan the official language of the country, and modern Italian became the main language spoken in Italy due to the growing use of radio, television, and newspapers. Russian also has undergone various reforms. Similarly, Chinese also has been reformed greatly in 20th century. Because of these, we choose English, French, German and Spanish to examine their historical changes in last two centuries. We choose Chinese to compare with the former four languages. The following gives a detailed account of how to select discourse connectives and extract their historical data for these languages. 


\subsection{English}

Figure 1 shows the hierarchy of semantic labels for discourse connectives. All clause relationships (i.e., semantic meanings) in PDTB3.0 can be classified into four main senses: Comparison, Contingency, Expansion and Temporal, and each main sense consists of smaller relations. The whole classification contains three levels: SENSE, CLASS and TYPE. Such annotation system was adopted in other languages such as French and German. We could classify discourse connectives in these language into four senses (i.e., Comparison, Contingency, Expansion and Temporal) and examine their relative historical changes in these languages.

In PDTB3.0, a discourse connective can be either explicit or implicit. An explicit discourse connective is realized in the form of lexical items, but an implicit discourse must be inferred from the adjacent discourse units. Most explicit discourse connectives are subordinate and coordinate conjunctions as well as discourse adverbials. The discourse connectives are mostly explicit in English, yet approximately $40 \%$ of the discourse connectives are implicit. In the present study, we will investigate the diachronic changes in frequencies of explicit discourse connectives. We can obtain 160 discourse connectives from PDTB3.0. The number of two-word phrase is 50, and that of three-word(or more) phrase is 20. Additionally, we get the most frequent 40 discourse connectives to make comparison.

However, we think it does not suffice to just obtain the historical frequencies of an individual discourse connective. As is well-known, a discourse connective can work in different discourse relations, that is, it can have different semantic meanings in the PDTB. It is difficult to obtain the data on the frequencies of these different meanings for a specific discourse connective. For example, the frequency of "although" was "158" (by one million words) in 1880s. However, the semantic meanings of "although" include contrast, concession, exception, and temporary. We want to know the frequency of those occasions where "although" is used with a contrast meaning or a concession meaning. In this case, the data from the PDTB3.0 manual can be used to calculate the weights of these different semantic meanings for a specific discourse connective. The following discusses how to calculate the frequency weights of different classes (semantic meanings) for the same discourse connective. All discussions about it be summarized into Table 1 in the supplementary materials (MS). In additionally, we can also classify disourse connectives into three types according to their orthography forms. For instance, "however" is monogram, and "because of" is bigram, while " as 

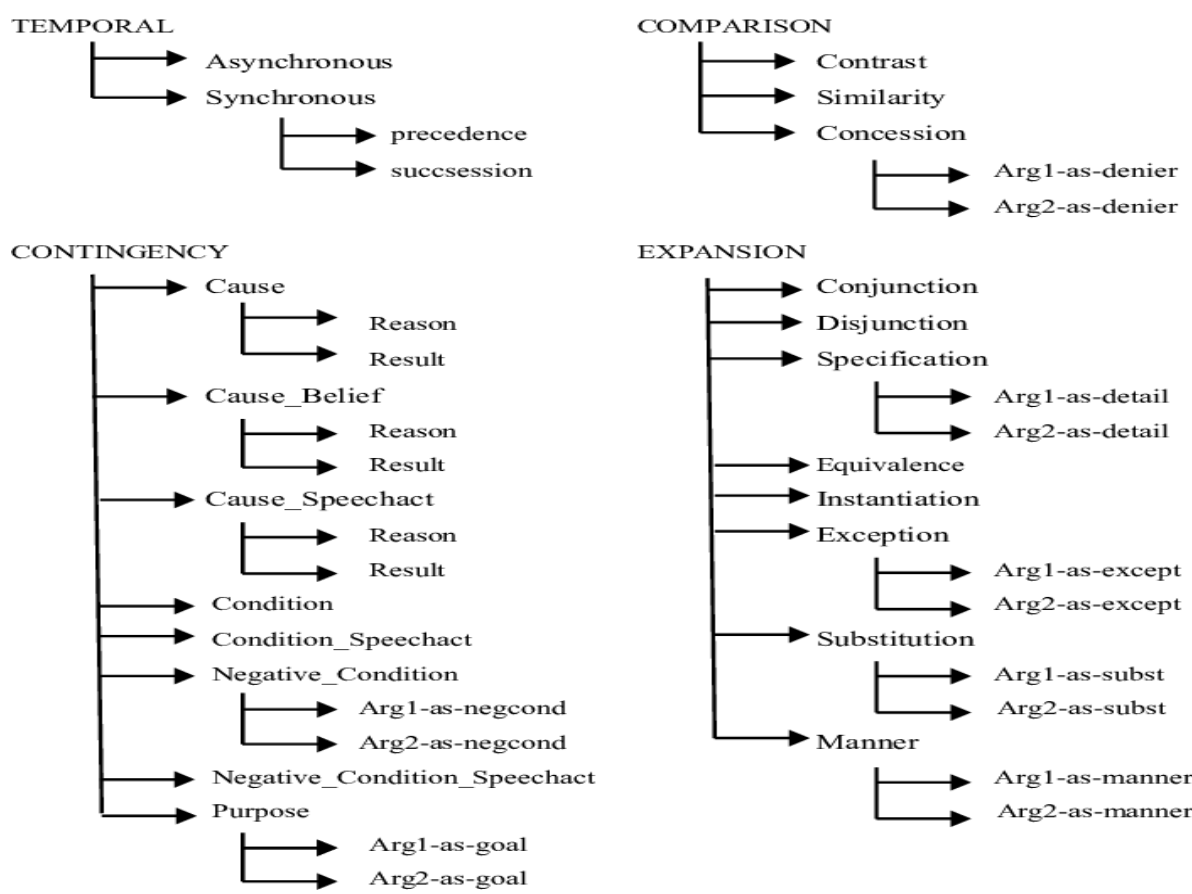

Figure 1: The hierarchical classification of clause relationships in PDTB3.0 (three levels: sense, class, and type)

soon as" is trigram.

English data on historical frequencies is derived from Google Books (Ngram) corpora (eng_2019) and the Corpus of Historical American English (COHA). We can obtain the data on historical frequencies from Google Books corpora, and we choose the period from 1800 to 2019. The two sub-corpora: English and English fiction. In order to cross-verify Google data, we also obtain the data on the historical frequencies of these discourse connectives from a large-scale historical English corpus. The COHA is a good candidate. This corpus consists of a number of balanced genres and represents realworld English language use over a long period of time. This corpus can provide the data on frequencies of an individual word by decade from 1820 to 2010. Parts of speech (PoS) of the majority of discourse connectives are conjunctives or prepositions. If a discourse connective has multiple PoSs, we will exclude those PoSs that cannot act as discourse connective. For example, when "like" works as verb, it cannot act as discourse connectives, so when we search the COHA, "verb" will be excluded. 


\subsection{French and German}

An inventory of most frequent discourse connectives ( $n=40$, e.g., "sondern", "weil", "inzwischen", "außerdem") in German can be obtained from the Potsdam contemporary corpus. However, a complete list of discourse connectives $(\mathrm{n}=135)$ can be obtained from Connective-Lex. info (Stede et al., 2019). In the two databases, the PDTB-style annotations were adopted, that is, we can classify these German discourse connectives into four senses which were taken in English. That means that we can examine historical changes of each sense of German discourse connectives. However, unfortunately, we only have to extract frequency data from the Google Ngram Viewer (ger_2019). There is not a historical corpus like COHA in German to provide historical data with us to cross-verify with each other.

Similarly, an inventory of most frequent discourse connectives $(n=40$, e.g., "et même", "ensuite", "comme pour") in French can be obtained from the ConcoLeDisCo corpus. Similarly, a complete list of discourse connectives $(n=175)$ can also be obtained from Connective-Lex. info. The PDTB-style annotations were adopted in the two French databases, suggesting that we can classify these French discourse connectives into four senses which were taken in English. Unfortunately, we only have to extract frequency data from the Google Ngram Viewer (fre_2019). There is not a historical corpus like COHA in French to provide historical data with us to cross-verify with each other.

However, as German and French discourse corpora do not provide a detailed account of frequencies on different classes of discourse connectives, we could not compute the weights of these different classes in English. Additionally, we just examine historical changes of each sense of discourse connectives in either German or French rather than those at the class level of discourse connectives.

\subsection{Spanish and Chinese}

We have not found any discourse corpora or databases in Spanish to extract a list of discourse connectives. We just find some relevant studies concerning discourse connectives in Spanish. Considering this, we just extract an inventory of the 40 most common discourse connectives rather than a complete list ( e.g., "por ejemplo", "en cambio", "de ahı")(Sáez, 2003)also from (https:// happylanguages.co.uk/lesson/spanish-connectives/), and frequency data can be extracted from the Google Ngram Viewer(spa_2019). 
We can extract a complete list of discourse connectives $(n=128)$ from CDTB Zhou and Xue (2015) for Chinese. In order to make comparison, we also extract 40 the most frequent ones in Chinese (e.g., “因为”, “不管”, “但是” ). The frequency data can be extracted from the Google Ngram Viewer(chn_sim_2019). We have to be aware of the fact that classical Chinese referring to the written language of the classical period of Chinese literature, was used from the end of the Han dynasty to the early 20th century, when it was replaced by vernacular written Chinese. Between 1920 to 1950, a mix use of vernacular written Chinese and Classical Chinese existed. The vernacular written form has been dominated after promoting the campaign of pǔtōnghuà (simplified Chinese, or "common speech") after 1955 (Norman, 1988). The last two centuries' history of Chinese is quite different from English, French, German and Spanish.

The following table overviews the materials we used in the current study.

Table 1: Languages, Items and Data Sources

\begin{tabular}{ccccccc}
\hline Language & $\begin{array}{c}\text { All Discourse } \\
\text { Connectives }\end{array}$ & $\begin{array}{c}\text { Most Frequent } \\
\text { Discourse connectives }\end{array}$ & $\begin{array}{c}\text { Discourse Connectives } \\
\text { Source }\end{array}$ & Four Senses & 15 Classes & $\begin{array}{c}\text { Frequency Data } \\
\text { Source }\end{array}$ \\
\hline \multirow{2}{*}{ English } & Yes $(\mathrm{n}=160)$ & Yes $(\mathrm{n}=40)$ & PDTB & Yes & Yes & Google Books Corpora \& COHA \\
English Fiction & Yes $(\mathrm{n}=160)$ & Yes $(\mathrm{n}=40)$ & PDTB & Yes & Yes & Google Books Corpora \\
French & Yes $(\mathrm{n}=175)$ & Yes $(\mathrm{n}=40)$ & $\begin{array}{c}\text { Connective-Lex.info, } \\
\text { Potsdam Corpus }\end{array}$ & Yes & No & Google Books Corpora \\
German & Yes $(\mathrm{n}=135)$ & Yes $(\mathrm{n}=40)$ & $\begin{array}{c}\text { Connective-Lex.info, } \\
\text { ConcoLeDisCo }\end{array}$ & Yes & No & Google Books Corpora \\
Spanish & No & Yes $(\mathrm{n}=40)$ & Relevant Studies & No & No & Google Books Corpora \\
Chinese & Yes $(\mathrm{n}=128)$ & Yes $(\mathrm{n}=40)$ & CDTB & No & No & Google Books Corpora \\
\hline
\end{tabular}

\section{Results}

\subsection{English}

First, we represented the historical changes in frequencies for discourse connectives extracted from Google Books Corpora and COHA respectively. The results from Google Corpora are shown in Figure 2. Figure 2 shows that English and English fiction underwent a significant decline in frequencies. In each case, we also examined all discourse connectives and the 40 most frequent ones. The regressive statistical analysis in each case shows that the slope is negative and the $\mathrm{p}$ value $(<0.001)$ is significant. This indicates that 
English users have significantly reduced their use of explicit discourse connectives over the last two hundred years. However, after 2000, the four cases began to increase dramatically. Overall, the four cases are greatly similarly in change patterns.

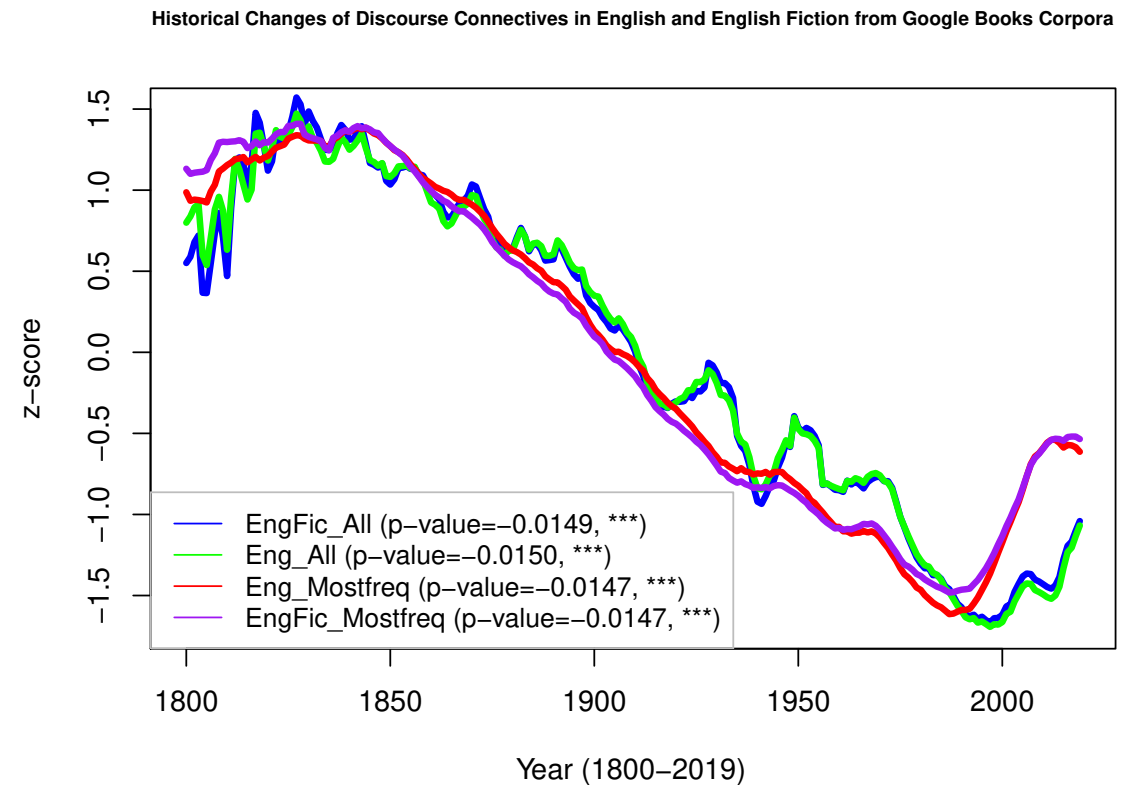

Figure 2: English and English fiction from Google Corpora have similar trends. Here y-axis represents scaled normalized frequency. Y-aixs in Figures 2-5 represents scaled frequency. p-value in the legend text represents the simple regression model result. Specifically, when p- value is smaller than 0.001, it is significant with three stars. Here "EngFic_All" represents all discourse connectives in English Fiction from Google Books Corpora. "EngFic_Mostfreq" represent the 40 most frequent discourse connectives in English Fiction from Google Books Corpora. "Eng_All" represent all discourse connectives in the English language from Google Books Corpora. "Eng_Mostfreq" represents the 40 most frequent discourse connectives of the English language from Google Books Corpora.

We represented the historical changes in frequencies for discourse connectives extracted from COHA. In each case, we also examined all discourse connectives and the 40 most frequent ones. The regressive statistical analysis in each case shows that the slope is negative and the p value $(<0.001)$ is significant, shown in Figure 3. We find that Figures 2 and 3 are completely consistent. That suggests that the data from Google is consistent from the one from COHA.

Moreover, we also represented the historical changes in frequencies for 


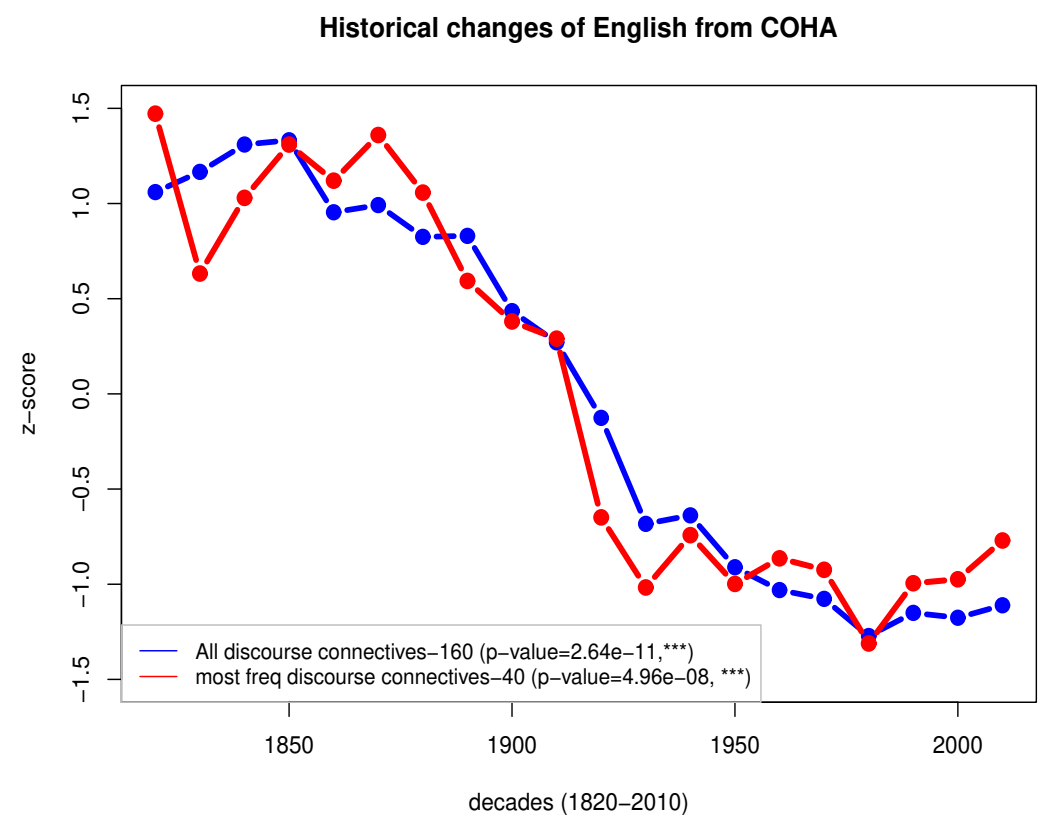

Figure 3: All English discourse connectives and the most frequent ones from COHA have similar trends. $\mathrm{x}$-axis represents year, and $\mathrm{y}$-axis represents the scaled frequency data. $p$-value in the legend text represents the simple regression model result. Specifically, when $p$-value is smaller than 0.001, it is significant with three stars. Here "All discourse connectives" represents all discourse connectives in the English language from the COHA. "Mostfreq discourse connectives" represents the 40 most frequent discourse connectives of the English language from the COHA.

all discourse connectives as classified into the four senses in Figure 4, which will be discussed in the following subsection. As discussed in the section of methods and materials, we examined 16 classes in English. Due to the space limit, we put the results in the supplementary materials. However, we can report the main findings here.

According to Asr and Demberg (2012), implicitness represents the proportion of implicit frequency to the total frequency. When a discourse relation with high implicitness, it indicates that language users tend to use explicit form of discourse connectives less frequently. For example, People seem to need more cues to help them understand adversative relations between clauses and sentences. That is why the relations of concession, condition and exception have low implicitness. Language users should not reduce the use of discourse relations with low implicitness. Otherwise, it will lead to incoherence and vagueness in discourse. However, Table 2 shows that such discourse relations have also reduced the use of explicit forms from a histor- 
ical perspective. Overall, the decrease of frequencies have been seen in the majority of 16 classes of English.

Table 2: Different types of relations in the PDTB3.0 and their historical changes

\begin{tabular}{ccc}
\hline Discourse relation classes & Implicitness & Diachronic trend \\
\hline Level-of-detail & $92.74 \%$ & down \\
Equivalence & $92.56 \%$ & down \\
Instantiation & $82.52 \%$ & up \\
Purpose & $80.91 \%$ & down \\
Cause & $73.96 \%$ & stable \\
Manner & $64.32 \%$ & down \\
Contrast & $44.34 \%$ & down \\
Asynchronous & $38.9 \%$ & down \\
Conjunction & $33.29 \%$ & down \\
Synchronous & $24.86 \%$ & down \\
Concession & $23.74 \%$ & down \\
Similarity & $23.31 \%$ & down \\
Exception & $12.5 \%$ & stable \\
Condition & $11.82 \%$ & down \\
Disjunction & $8.96 \%$ & down \\
\hline
\end{tabular}

\subsection{French, German and Spanish}

We put English, French and German together to find their similarity of the change patterns. There are six different cases. First, we examined changes by looking at all discourse connectives and the 40 most frequent ones in these languages. Second, we examined changes of frequencies of discourse connectives in terms of four senses of discourse relations by the PDTB style. This panel is completely consistent with Figures 1 and 2 .

As shown in Figure 4, the panel of "most frequent" shows the historical changes of 40 most frequent discourse connectives in English, French, German and Spanish over the last two centuries. This panel shows the great similarity among the four languages. The panel titled with "All discourse connectives" shows the frequency changes of all discourse connectives in English, French and German. The other four panels titled with Comparison, Contingency, Expansion and Temporary examine the four different types 

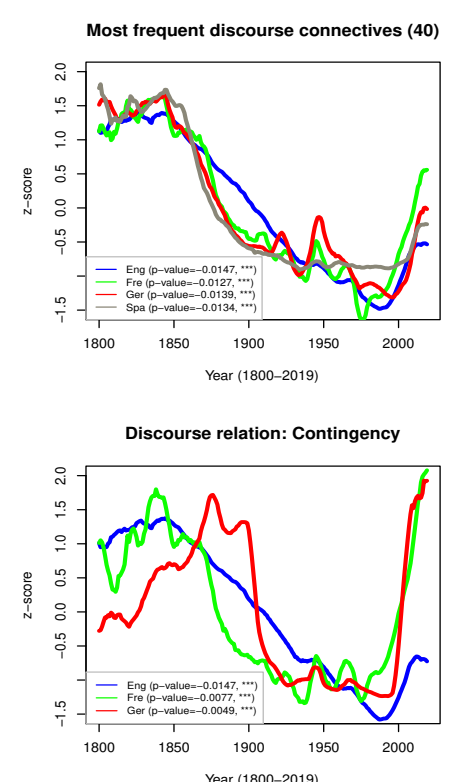

of discourse connectives (by PDTB) over the last two centuries in English, French and German. These five panels also see the general similarity pattern: first, the simple regression model shows all of the cases have a significant decline (p-value is smaller than 0.001). Second, at the initial stage (1800-1850), English, French and German saw an increase in frequencies. However, after 1850, the decline was obvious. By contrast, after 2000, all cases have seen a rise. Nevertheless, after 2000, the increase in French and German has been more remarkable than English.
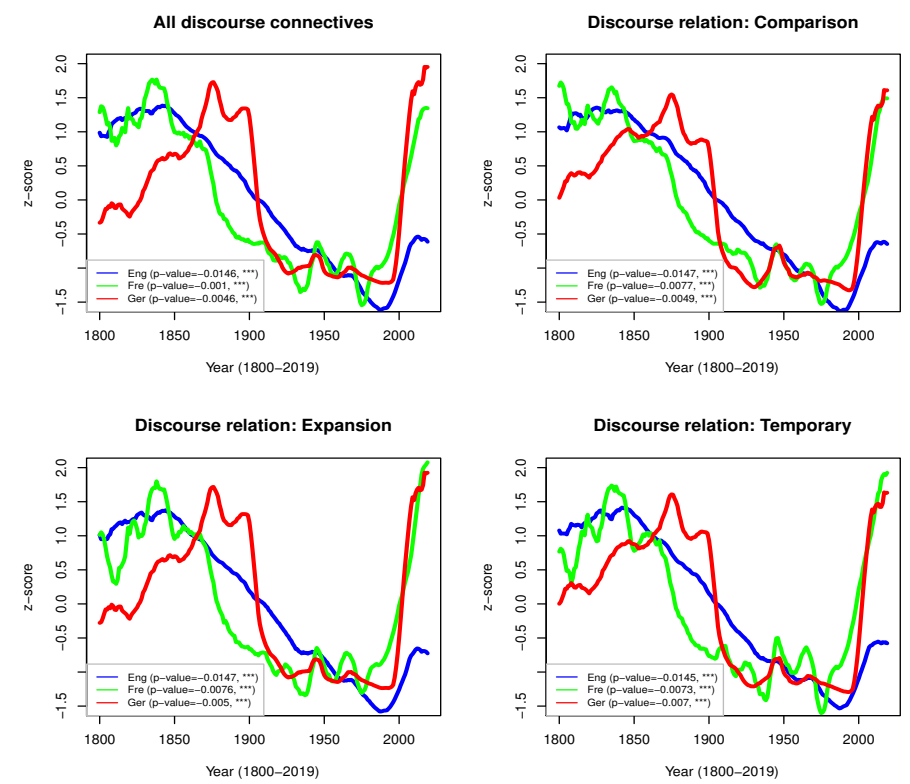

Figure 4: Four languages show similar trends in different cases. $p$-value in the legend text represents the simple regression model result. Specifically, when $p$ value is smaller than 0.001, it is significant with three stars. Eng=English, Fre=French, Ger $=$ German .

\subsection{Chinese}

The changes of Chinese have a perfect fit to historical events. As mentioned in section of methods and materials, the modern written form of Chinese was dominated after 1955. The period of 1920 and 1955 saw the mixed use of modern Chinese and classical Chinese. Before 1920, the use of classical Chinese was dominant in written form. The left panel in Figure 5 clearly show such changes. We used discourse connectives from modern Chinese to explore how their frequencies changed. During the period of 1920 and 


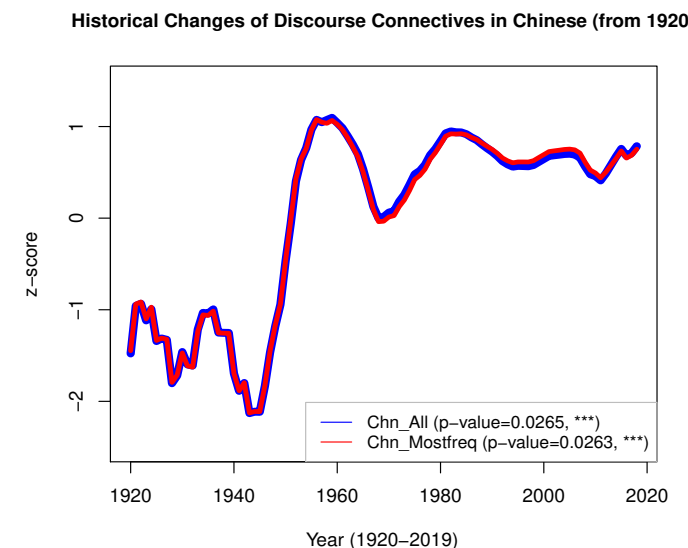
increased dramatically after 1950 .
Historical Changes of Discourse Connectives in Chinese (from 1950)

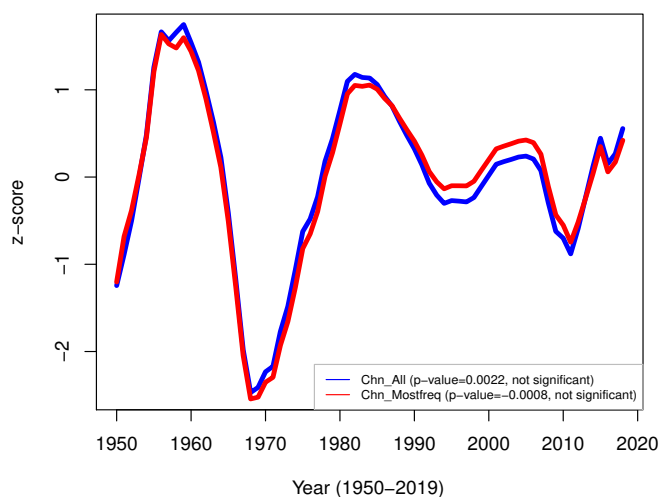

Figure 5: Historical changes of discourse connectives in Chinese. "Chn_All"=All discourse connectives in Chinese, "Chn_mostfreq" = the 40 most frequent discourse connectives in Chinese. The time span in the left panel is from 1920 to 2009, and that in the right panel is from 1950 to 2009 .

1950, the use of modern Chinese discourse connectives began to increase but

However, such an increase began to drop quickly after 1960 because of the "cultural revulotion" and a series of political campaigns in China. The books published during the period were likely to propaganda the leader's thoughts, ignoring the coherence and logical connection in them, and this could be reflected in the right pannel of Figure 5. After the "cultural revulotion" ended (1976), the frequencies of discourse connectives increased dramatically. In 1980s, the changes kept stable. The difference is that after 2000, the frequencies have been fluctuated relatively stably. By contrast, an more obvious increase has been seen in English, French, German and Spanish after 2000 .

\section{Discussion}

It would be easy to accept that the frequencies in explicit discourse connectives concerning contingency declined over the course of time because the causality-by-default hypothesis supports the view that people do not need extra cues in understanding causality. For instance, Jin and de Marneffe (2015) proposed that expansion should be treated as being the default option as it is explicitly marked far more often than causation in English. It is 
clear that causation is not the least marked relation. We can also see that contingency and comparison are often implicit. Furthermore, many other language devices are capable of replacing such discourse connectives. For example, causality verbs can indicate the causal relation between two sentences. However, our finding on historical changes of adversative connectives (contrast, concession) seem to contradict our intuition. People seem to need more cues to help them understand adversative relations between clauses and sentences. Despite this, our data supports that language users tend to be historically less reliant on the use of adversative connectives. Additionally, the downward trend of discourse connective is not limited in some specific genres, and it can be found in academic genre. Biber and Gray (2010) found there is also evidence that this style of discourse is spreading to a less explicit marking of meaning relations generally in academic texts. They found that linking adverbials were quite common in academic writing in the 18th and 19 th centuries, but they have decreased rapidly in use in the 20th century. The finding is consistent with the one in the current study.

As mentioned in the section of introduction, the thesis of "the Rise of Discourse Markers" has gradually become accepted. This nonetheless gives rise to the illusion that the use of discourse connectives has increased and thus that discourse connectives have risen in frequency. However, our data demonstrates that the frequencies of discourse connectives have been declined significantly over more than two hundred years. However, the rise of them can be seen from 1980. Given the overall change and the recent change of their frequencies, we cannot support the hypothesis of a rise of discourse markers from a historical perspective.

The following will discuss the relevant issues more broadly so that we can gain bigger picture. Scheffer et al. (2021) reported an historic rearrangement, occurring in the late 20th century, of the balance between reason and emotion. Note that their study just focuses on the period between 1850 and 2019. Its approach is part of a new trend in the psychological sciences that uses extremely large volumes of text to study basic patterns of human cognition. Such methods have been used in exploring the universal properties of gender representations (Charlesworth et al., 2021), the rise of causal thinking (Iliev and Axelrod, 2016) and a cognitive bias towards positivity in language itself (Dodds et al., 2015). The current study can also be associated with these previous researches and contributes to the knowledge of human cognitive changes in history.

Scheffer et al. (2021) found a shift from the intuitive pole to the rational pole (the "rise" of rationality) and then back (the "fall"). The rise has 
begun by the start of their data in 1850, and unfolds over the course of a century or more. The fall begins in 1980, and is more rapid than the rise: by 2020 , the balance is similar to that seen in the early 1900s. The fall appears to accelerate in the early 2000s. By contrast, Scheffer et al. (2021) found sentiment words and intuition related words have undergone the reversed process. They began to fall from 1850 but rose from 1980 but increased quickly after 2000s. Interestingly, the changes of emotion and intuition words are consistent with the ones of discourse connectives in the current study. We wonder why this coincidental change happened.

The distinction between rational words and emotional (intuition) words can roughly be taken as the difference between formal words and informal words, that is, language style difference. In terms of Scheffer et al. (2021), rational words are related to rationality, science, and quantification. These rational words are actually used in formal situations, particularly in writing for professional or academic purposes. Emotional words concerned with intuition, believing, and spirituality are more likely used in informal circumstances than formal ones (e.g.,imagine-envisage, fear-apprehension). We collected 210 pairs of formal words and their corresponding informal words (https://engdic.org/formal-and-informal-words/). After having obtaining their historical frequencies from Google Books corpora (1850-2019), we used the second principal component (PC2) shows an asymmetric U-curve in terms of the method by Scheffer et al. (2021). Shown in Fig.6, the historical change trend of formal words is basically identical with that of rational words. The same are with informal words and emotional words.

The historical changes of formal/informal words are in fact in line with the trend of "colloquialization". Recent numerous studies found that "colloquialization" has become a trend in the development of language (Leech, 2004). Mair (2006):187 proposed that "a significant stylistic shift in twentieth century English" is due to which the written language becomes more similar to the spoken language and more tolerant to various degrees of informality. Overall, colloquialization is able to explain these changes in Scheffer et al. (2021). This simpler approach can be used to explain the phenomenon of language change more effectively.

Similarly, the colloquialization trend is well capable of explaining the changes of discourse connectives. When the written language becomes more similar to the spoken language, language speakers tended to use less discourse connectives. The other evidence could also prove it. Recent years saw the fact that the sentence length is gradually becoming shorter in history (Liberman, 2011; Rudnicka, 2018; Sun and Wang, 2019). It is known that discourse 

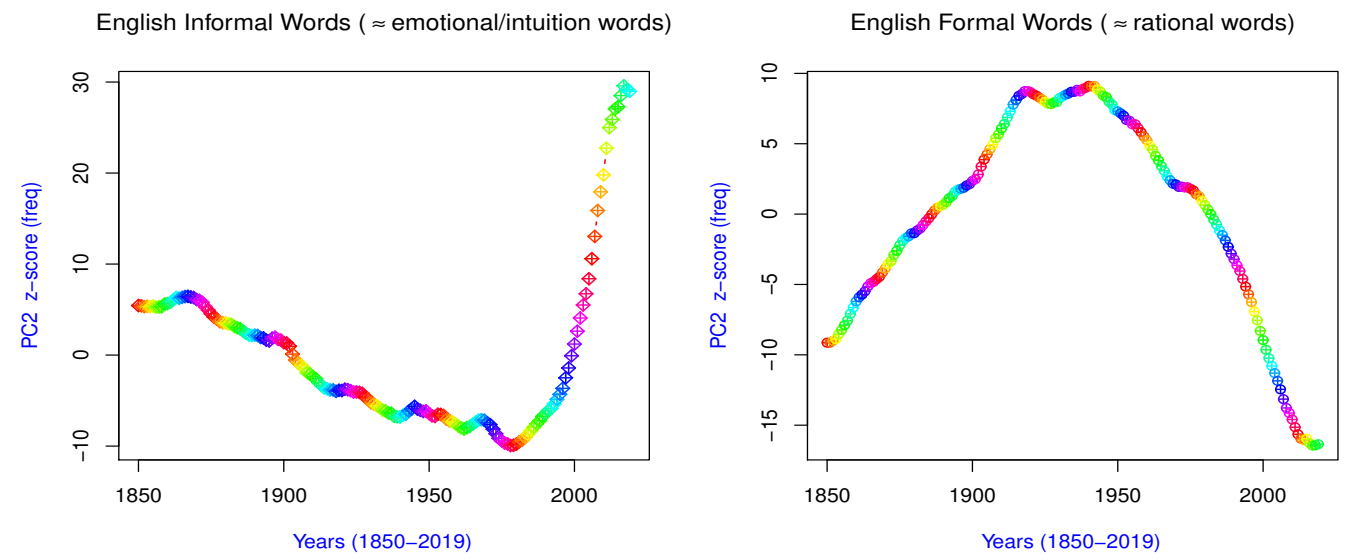

Figure 6: Historical changes of informal words and formal words in English. $\mathrm{y}$-axis represents second principal component of change in z-scores of frequencies.

connectives are mainly used to connect between clauses and sentences. The shorter length of sentence indicates that people have used less discourse connectives. The trend of using shorter sentence can be found in French, German and Spanish. However, the trend has kept stable since 2000s, which could be verified in Google N-gram viewer using period, exclamation mark, question mark and colon. It means that the sentence length change is consistent with the frequency change trend of discourse connectives. However, using shorter sentence length is a result of colloquliaztion. It is a tendency for the written language gradually to acquire norms and characteristics associated with the spoken conversational language. This trend is the main motivation to the decrease of discourse connectives.

The other possibility of using less discourse connectives is that some similar expressions in language could have replaced specific discourse connectives. For example, Iliev and Axelrod (2016) found an increase in the use of causal language ("causality") from 1800 to 2000. The causality expressions in Iliev and Axelrod (2016) also includes some contingency discourse connectives, but most of them are verbs and nouns. The data from Iliev and Axelrod (2016) shows that there is an decrease from 1980s but a quick drop has been seen after 2000. It could be possible that some causality expressions in language could have replaced some discourse connectives used for contingency. This example can support our argument that discourse connectives could be partly replaced by some Note that the function causality within a sentence does work as causality discourse connectives. A causality discourse 
connective could impact several clauses even a text (e.g., "because", "consequently"). By contrast, intra-sentential causality expressions (e.g, "enable", "prevent") just influence one sentence. In short, the two types of causality expression are distinct. From the data from Iliev and Axelrod (2016), intrasentential causality has been increasing from 1800 to 2000. This does not conflict with our finding on a decease of contingency discourse connectives. When intra-sentential causality was used more frequently, the less frequently the contingency discourse connectives could occur. Clearly, "causality" is treated as one type of rationality in language. It could explain why "causality" increased but contingency discourse connectives decreased.

When language users are historically less reliant on the use of discourse connectives, we need to consider whether this has led to incoherence in discourse more seriously or not. However, the colloquialization trend allows language user to introduce other different expressions to compensate this gap.

In the present study, in German and French, the trends in discourse connectives suddenly accelerate around 2000. One possible explanation could be that the standards for inclusion in " to "from a publisher that directly deposited with Google" after 2004 to 2007. It means that from 2004, more reprinted books could have added to ngram corpora, so that some expressions frequencies accelerated (Schmidt et al., 2021). That is why some discourse connectives have rocketed after 2004. However, such a trend is not so strong in English as German and French.

We have discussed the possible factors to cause an decease of discourse connectives from different perspectives. The following will discuss what more factors cause language change from broader perspectives. Many studies show that word correlations, occurring across time and between words, are largely influenced by coevolutionary social, technological, and political factors, also including population size, literary education and other factors (Koselleck, 1989; Wichmann and Holman, 2009; . The impact of historical context on language dynamics is not limited to emerging languages, but extends to languages that have been active and evolving continuously. For example, Changes of discourse connectives in Chinese was greatly influenced by historical events and broader social-cultural changes. This provides a perfect example to illustrate the co-evolution of social, political factors with language (Petersen et al., 2012; Jablonka et al., 2012; Sun et al., 2021). Additionally, we find that the distribution of discourse connectives growth rates significantly broadens during times of balance among different factors, revealed through English, French, German and Spanish during the last two centuries. 
The recent peak in German and French is almost as high as that 150 years ago. This can be understood as manifesting from the unification of public interests over the last 150 years.

\section{Conclusion}

This study made historical corpora to examine diachronic changes of discourse connectives in Chinese, English, French, German and Spanish. We found that there has been a clear decline of frequencies of these discourse connectives in English, French, German and Spanish. More interestingly, an increase of frequencies can be seen after 1980s, and such increase facilitated after 2000s among the five languages. In the present study, our diachronic data on frequency in discourse connectives does not support the hypothesis of rising of discourse connectives mentioned in Introduction. We believe that the diachronic changes of discourse connectives have been mainly influenced by the trend of "colloqualiation". The other factors also contributed to this language phenomenon. The current study reveals a cultural and human behavior trend analysis based around changes of discourse connectives, and it provides novel insights into the development of human society.

\section{References}

Asr, F. T. and Demberg, V. (2012). Implicitness of discourse relations. In the Proceedings of COLING 2012, pages 2669-2684.

Berry, D. M. (2011). The computational turn: Thinking about the digital humanities. Culture Machine, 12.

Biber, D. and Gray, B. (2010). Challenging stereotypes about academic writing: Complexity, elaboration, explicitness. Journal of English for Academic Purposes, 9(1):2-20.

Charlesworth, T. E., Yang, V., Mann, T. C., Kurdi, B., and Banaji, M. R. (2021). Gender stereotypes in natural language: Word embeddings show robust consistency across child and adult language corpora of more than 65 million words. Psychological Science, 32(2):218-240.

Claridge, C. (2013). The evolution of three pragmatic markers: As it were, so to speak/say and if you like. Journal of Historical Pragmatics, 14(2):161184. 
Dodds, P. S., Clark, E. M., Desu, S., Frank, M. R., Reagan, A. J., Williams, J. R., Mitchell, L., Harris, K. D., Kloumann, I. M., Bagrow, J. P., et al. (2015). Human language reveals a universal positivity bias. Proceedings of the National Academy of Sciences, 112(8):2389-2394.

Ellis, N. C. (2002). Frequency effects in language processing: A review with implications for theories of implicit and explicit language acquisition. Studies in Second Language Acquisition, 24(2):143-188.

Fraser, B. (1999). What are discourse markers? Journal of Pragmatics, 31(7):931-952.

Grainger, J. (1990). Word frequency and neighborhood frequency effects in lexical decision and naming. Journal of Memory and Language, 29(2):228244.

Heine, B., Kaltenböck, G., Kuteva, T., and Long, H. (2022). The Rise of Discourse Markers. Cambridge University Press.

Iliev, R. and Axelrod, R. (2016). Does causality matter more now? increase in the proportion of causal language in english texts. Psychological Science, 27(5):635-643.

Jablonka, E., Ginsburg, S., and Dor, D. (2012). The co-evolution of language and emotions. Philosophical Transactions of the Royal Society B: Biological Sciences, 367(1599):2152-2159.

Jin, L. and de Marneffe, M.-C. (2015). The overall markedness of discourse relations. In Proceedings of the 2015 Conference on Empirical Methods in Natural Language Processing, pages 1114-1119.

Köhne-Fuetterer, J., Drenhaus, H., Delogu, F., and Demberg, V. (2021). The online processing of causal and concessive discourse connectives. Linguistics, 59(2):417-448.

Koselleck, R. (1989). Linguistic change and the history of events. The Journal of Modern History, 61(4):650-666.

Leech, G. (2004). Recent grammatical change in english: data, description, theory. In Advances in Corpus Linguistics, pages 61-81. Brill.

Lenk, U. (1998). Discourse markers and global coherence in conversation. Journal of Pragmatics, 30(2):245-257. 
Lenker, U. and Meurman-Solin, A. (2007). Connectives in the History of English, volume 283. John Benjamins Publishing.

Liberman, M. (2011). Real trends in word and sentence length. Online at $<$ http://languagelog. ldc. upenn. edu/nll.

Mair, C. (2006). Twentieth-century English: History, variation and standardization. Cambridge University Press.

Michel, J.-B., Shen, Y. K., Aiden, A. P., Veres, A., Gray, M. K., Team, G. B., Pickett, J. P., Hoiberg, D., Clancy, D., Norvig, P., et al. (2011). Quantitative analysis of culture using millions of digitized books. Science, 331(6014):176-182.

Norman, J. (1988). Chinese. Cambridge University Press.

Petersen, A. M., Tenenbaum, J., Havlin, S., and Stanley, H. E. (2012). Statistical laws governing fluctuations in word use from word birth to word death. Scientific Reports, 2(1):1-9.

Prado, J., Spotorno, N., Koun, E., Hewitt, E., Van der Henst, J.-B., Sperber, D., and Noveck, I. A. (2015). Neural interaction between logical reasoning and pragmatic processing in narrative discourse. Journal of Cognitive Neuroscience, 27(4):692-704.

Rudnicka, K. (2018). Variation of sentence length across time and genre. Diachronic corpora, genre, and language change, pages 220-240.

Rysová, M. (2017). Discourse connectives: From historical origin to presentday development. New Perspectives on Cohesion and Coherence, page 11.

Sáez, F. T. (2003). Culture in writing: discourse markers in english and spanish student writing. Tadea seu liber de Amicitia, pages 345-364.

Sanders, T. J. and Noordman, L. G. (2000). The role of coherence relations and their linguistic markers in text processing. Discourse Processes, $29(1): 37-60$.

Scheffer, M., van de Leemput, I., Weinans, E., and Bollen, J. (2021). The rise and fall of rationality in language. Proceedings of the National Academy of Sciences, 118(51).

Schmidt, B., Piantadosi, S. T., and Mahowald, K. (2021). Uncontrolled corpus composition drives an apparent surge in cognitive distortions. Proceedings of the National Academy of Sciences, 118(45). 
Stede, M., Scheffler, T., and Mendes, A. (2019). Connective-lex: A webbased multilingual lexical resource for connectives. Discours. Revue de Linguistique, Psycholinguistique et Informatique. A Journal of Linguistics, Psycholinguistics and Computational Linguistics, (24).

Sun, K. and Baayen, R. H. (2021). Hyphenation as a compounding technique in english. Language Sciences, 83:101326.

Sun, K., Liu, H., and Xiong, W. (2021). The evolutionary pattern of language in scientific writings: A case study of philosophical transactions of royal society (1665-1869). Scientometrics, 126(2):1695-1724.

Sun, K. and Wang, R. (2019). Frequency distributions of punctuation marks in english: Evidence from large-scale corpora. English Today, 35(4):23-35.

Torabi Asr, F. and Demberg, V. (2020). Interpretation of discourse connectives is probabilistic: evidence from the study of but and although. Discourse Processes, 57(4):376-399.

Van Silfhout, G., Evers-Vermeul, J., and Sanders, T. (2015). Connectives as processing signals: How students benefit in processing narrative and expository texts. Discourse Processes, 52(1):47-76.

Webber, B., Prasad, R., Lee, A., and Joshi, A. (2019). The penn discourse treebank 3.0 annotation manual. Philadelphia, University of Pennsylvania.

Wichmann, S. and Holman, E. W. (2009). Population size and rates of language change. Human Biology, 81(3):259-274.

Zhou, Y. and Xue, N. (2015). The chinese discourse treebank: A chinese corpus annotated with discourse relations. Language Resources and Evaluation, 49(2):397-431.

\section{Supplementary Materials}


Table 3: Example of calculating frequency weights for differfor different PDTB classes of this discourse connective

\begin{tabular}{llll}
\hline discourse connective & sense & class & frequency \\
\hline Although & Comparison & Concession.Concession.Arg1-as-denier & 206 \\
Although & Comparison & Concession.Arg2-as-denier & 105 \\
Although & Comparison & Contrast & 104 \\
Although & Expansion & Exception.Arg2-as-execpt & 2 \\
Although & Temporal.Synchronous | Comparison.Contrast & $1(0.5+0.5)$ \\
\hline
\end{tabular}

$$
\Downarrow
$$

Table 4: weights for different PDTB classes of this discourse connective

\begin{tabular}{lll}
\hline discourse connective & sense.class & weight \\
\hline although & Comparison.Concession & $(206+105) / 311=0.9482$ \\
although & Comparison.Contrast & $(14+0.5) / 311=0.04421$ \\
although & Expansion.Exception & $2 / 311=0.0061$ \\
although & Temporal.Synchronous & $0.5 / 311=0.0015$ \\
\hline
\end{tabular}

\section{A Weights on different classes for discourse connectives}

The total frequency for "although" is 311 (per one million words), as shown in Table 3. We merge the items shared with the same sense and class regardless of types. For example, the first and second "although" are merged to be treated as "Comparison.Concession". The last "although" is annotated as "Temporal.Synchronous|Comparison.Contrast". In this case, the two types of "sense.class" will be split with equal frequency. Here, we split it into "Temporal.Synchronous" and "Comparision.Contrast" with the frequency of 0.5 respectively. Generally, "although" can be classified into four types according to its "sense.class". Each item has their own different frequencies. We are interested in the weight of each item. However, we have obtained the frequency for "Comparison. Constrast", so the frequency on this item (i.e. 0.5 ) is put into the one above. The weight of an individual "Sense.Class" is calculated by its frequency divided by the total frequency. For example, the weight of "Comparison.Concession" can be computed by its frequency $(206+105)$, divided by the total frequency (311), that is, its weight is 0.9482 . The data on the weights of the four different meanings 
(Sense. Class) is shown in Table 4.

The total frequency of "although" for each decade can be obtained in COHA. However, we are more interested in knowing the frequency of each type of "sense.class" for this word. It can be easily calculated using the equation of "the total frequency * the weight". For example, the total frequency (per million words) of "however" in 1900 is 378.76 . In this case, its frequency of "Comparision.Concession" is $378.76 * 0.9481707=359.13$, the one of "Comparision.Concession" is $378.76 * 0.04420732=16.74$, the one of "Expansion. Exception" is $378.76^{*} 0.0060975=2.31$, the frequency of "Temporal.Synchronous" is $378.76^{*} 0.001524=0.58$.

Using COHA, we can gain data on the frequencies (standard frequency, by one million words) of all discourse connectives derived from PDTB3.0 from 1810 to 2010. An individual discourse connective can be classified into several types according to the PDTB3.0 annotation manual, as shown in Table 1. We can calculate their frequencies in each decade using the corresponding frequency weights of the different classes for the same discourse connective (1820-2010). Note that the weights of the different classes of this discourse connective are based on the PDTB3.0, which used WSJ texts published in 1990s. That means these weights only reflect usage in the 1990s. However, the weights must have been somewhat different in each decade of the last two centuries. Nonetheless, the semantic changes in these discourse connectives are much smaller than nouns, verbs, and adjectives. The reason for this is that the majority of discourse connectives are conjunctions and prepositions. It is well-known that function words undergo a lesser degree of historical semantic changes than content words (Kutuzov et al., 2018). Bloomfield (1933) defines function words as "innovations which change the lexical meaning rather than the grammatical function of a form." . In view of the fact that these small semantic diachronic changes in these discourse connectives have been basically ignored, we maintain that the weights based on the PDTB can be used to calculate the frequencies over all decades. This can be done if we use the same weights for each decade between 1810 and 2010 

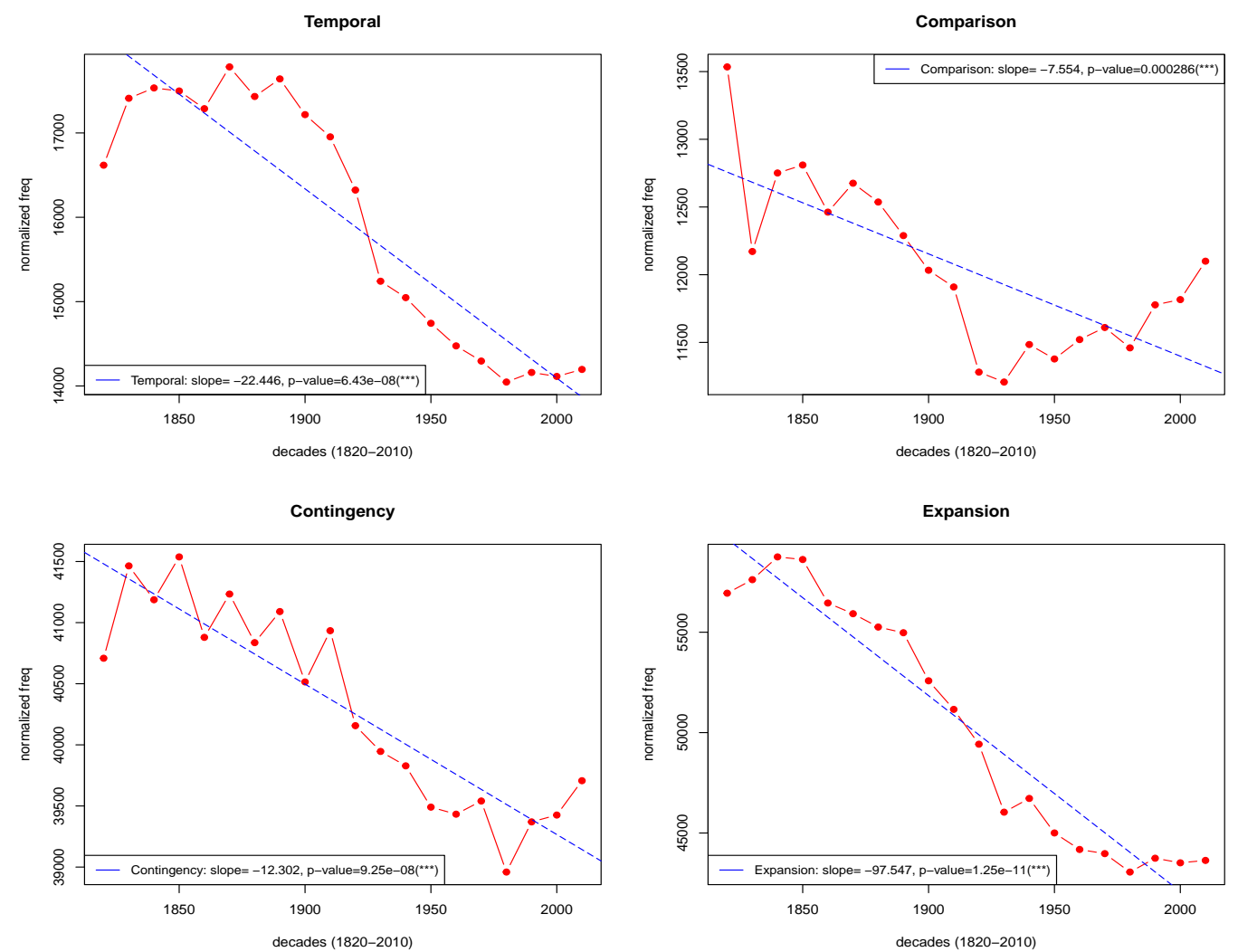

Figure 7: Changes of four senses from COHA
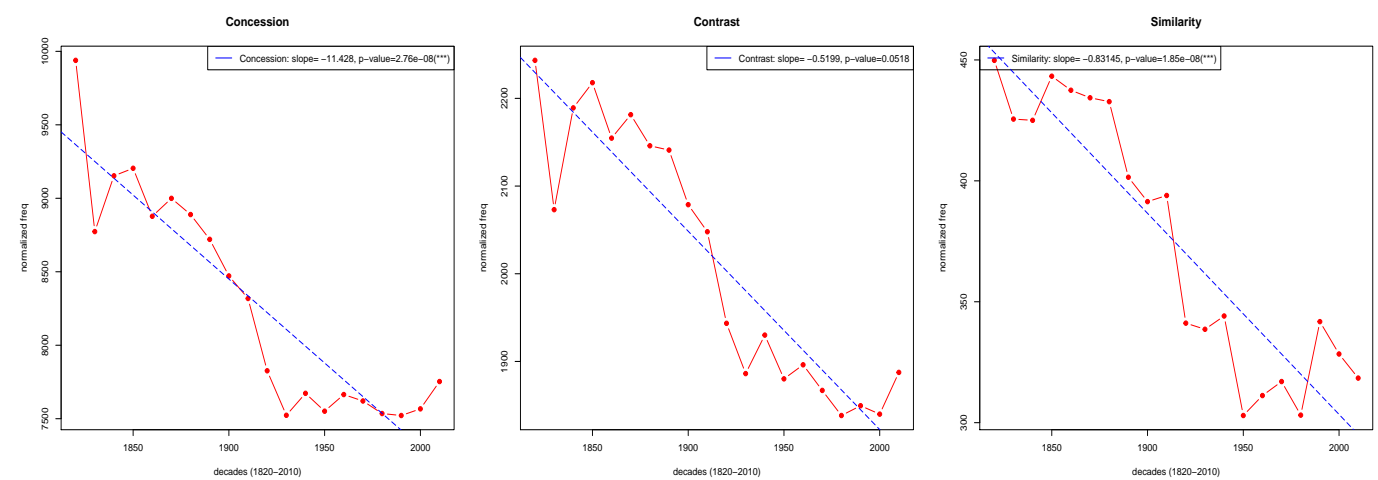

Figure 8: Changes of comparison classes from COHA

\section{B Historical changes of different classes in English from COHA}

The four classes under "expansion" show a significant decline in their frequencies. The linear regressive analysis (negative slopes and significant p- 

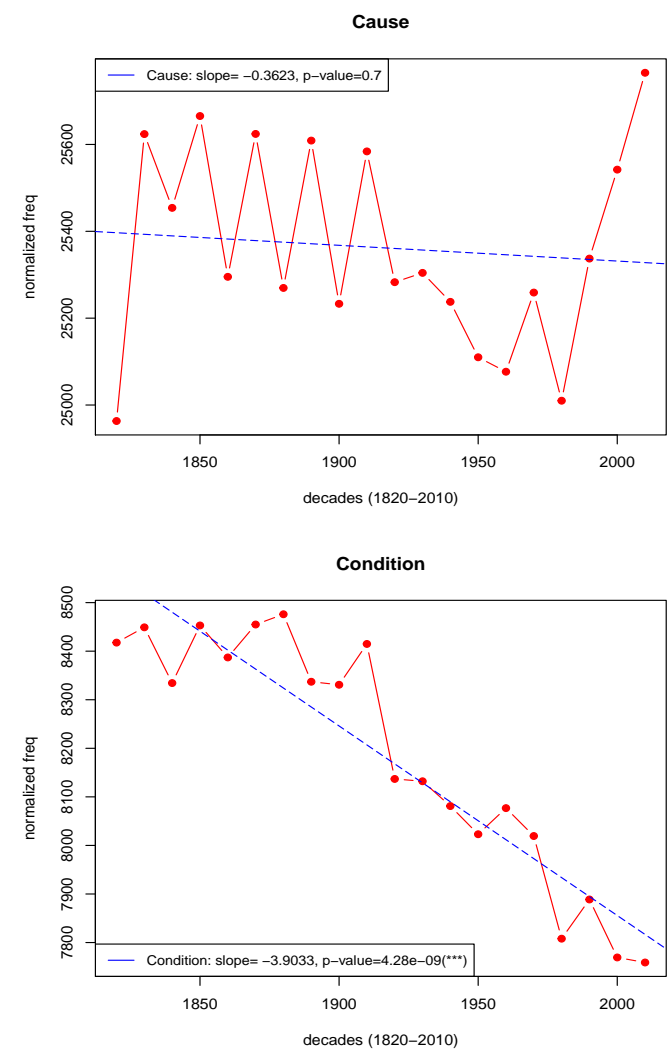
express comparative meanings.
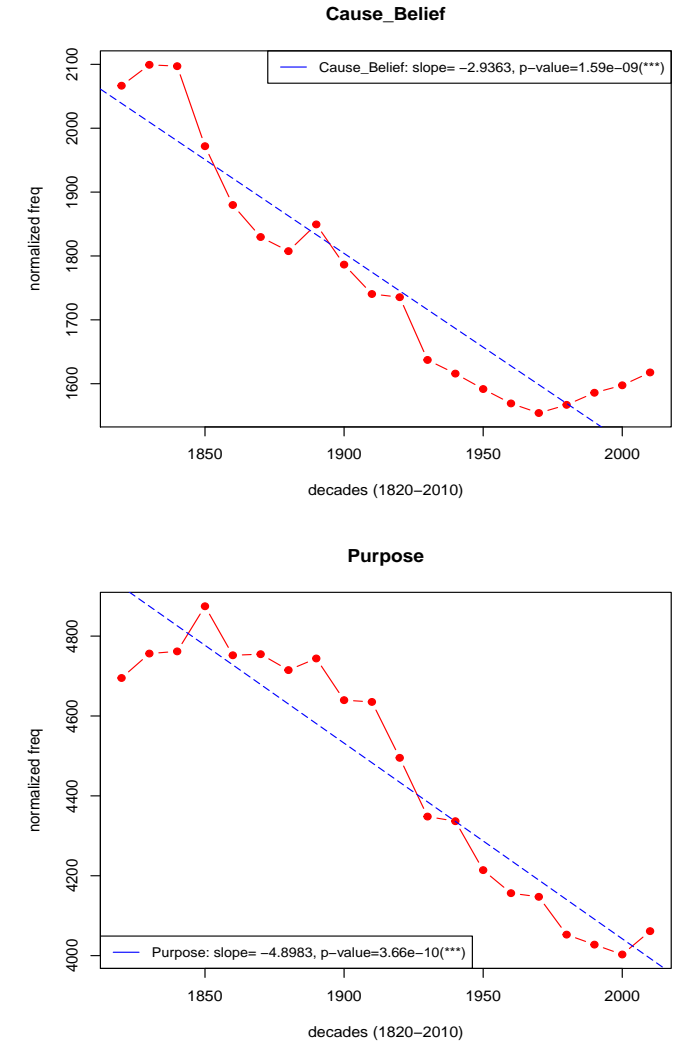

Figure 9: Changes of contingency classes from COHA

value $<0.001)$ also confirm this observation. By contrast, the increase in the frequency of "instantiation" may suggest that English users have preferred to use more examples. This preference leads to an increase in the use of discourse connectives involved in exemplification. The exception is "exception". The regression analysis shows that there is no significant change in this case. It indicates that the frequency of "exception" remains relatively stable.

What is very interesting is that the three classes under the category "comparison" show different trends. As is well-known, the proportion of explicit discourse connectives regarding "comparison" is relatively high. In other words, language users usually have to employ discourse connectives to

As shown in Figure 9, the frequency of the three classes shows a clearly declining trend. Due to the need for discourse connectives, the declining frequency of "concession" could indicate that language users tend to employ less concession-relationship clauses over the course of time. The regression 

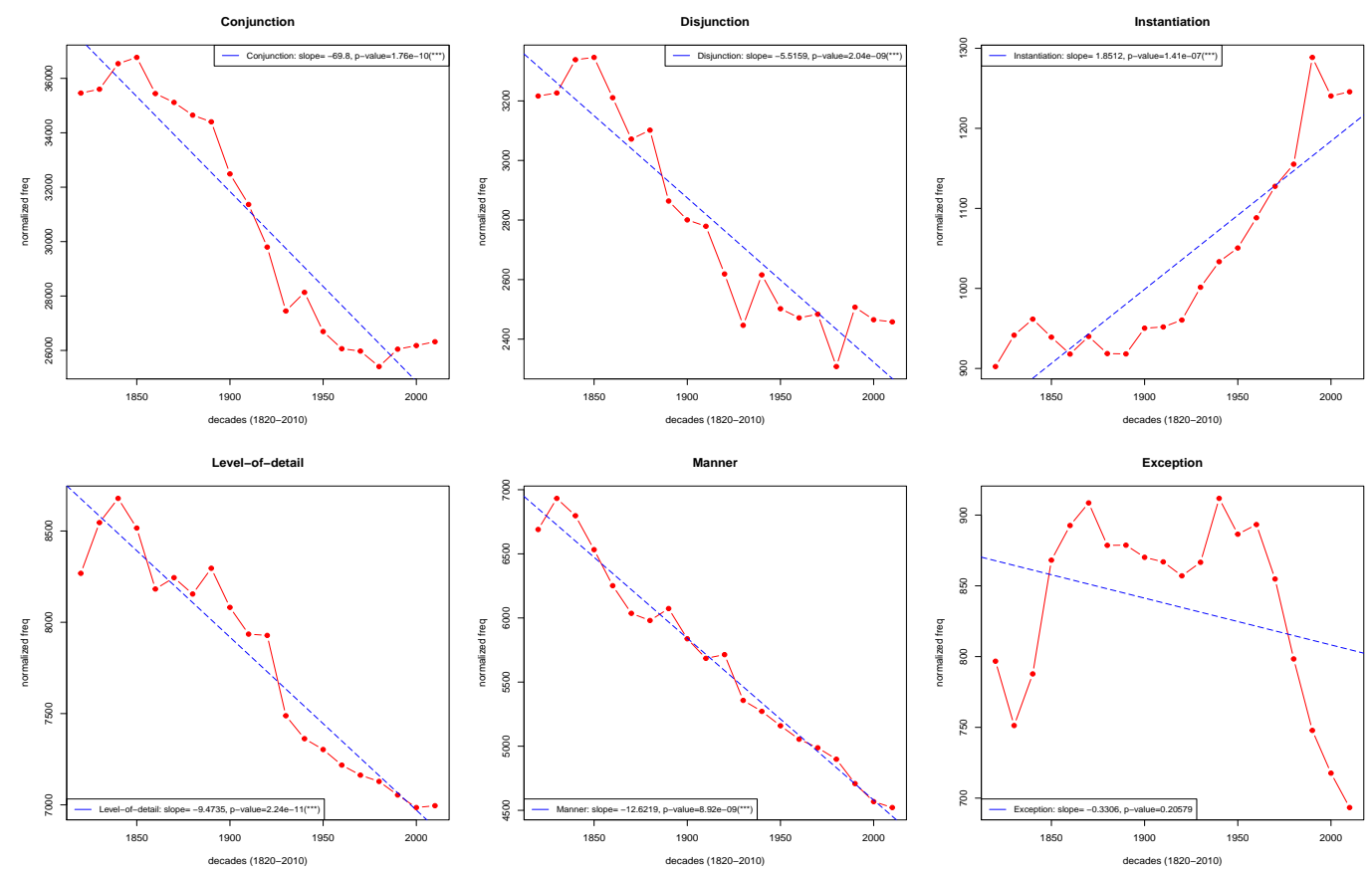

Figure 10: Changes of expansion from COHA

analysis shows that the p-value is greater than 0.05 , which suggests there is no dramatic changes in frequencies of contrast.

In the following, we examine the frequency changes from an orthographic perspective. As is well-known, some discourse connectives are just one word, but the others may consist of two or three (or more) words. "N-gram" here means one word, a two-word combination or a three-(or more)-words combination. Figure 10 represents the diachronic changes in different types of discourse connective from the perspective of n-gram.

Here "Bigram" represents two-word phrase such as "due to", "in fact". Trigram refers to three (or more than three) word phrases, such as "in any case", "on the other hand". "Lemma" refers to one-word discourse connectives (e.g., "similarly", "nevertheless"). Only Bigram shows an increasing trend in frequency. This could mean that English users tend to use two-word discourse connectives more frequently although the overall trend is a less frequent use of discourse connectives. 

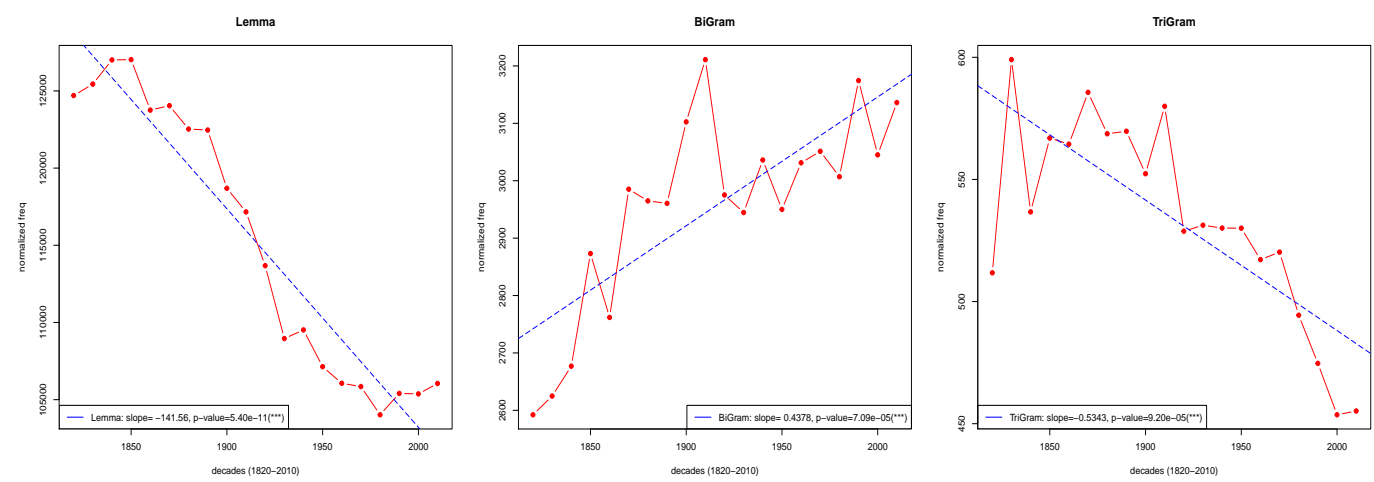

Figure 11: Four languages show similar trends in different cases 